\title{
The Supersaturation and Transient Volume Measurement for Nucleation, Growth, Coarsening in a Concentrated Ni-Based Superalloy
}

\author{
Sung-Il Baik, ${ }^{1,2}$ Zugan Mao, ${ }^{1,2}$ Elizaveta Y. Plotnikov, ${ }^{1}$ Ronald D. Noebe ${ }^{3}$, David N Seidman ${ }^{1,2}$ \\ 1. Department of Materials Science and Engineering, Northwestern University, Evanston, IL, USA \\ 2. Northwestern University Center for Atom Probe Tomography (NUCAPT), Evanston, IL, USA \\ 3. NASA Glenn Research Center, Cleveland, OH, USA
}

These Ni-based superalloys are strengthened by coherent ordered $\mathrm{Ni}_{3} \mathrm{Al}\left(\mathrm{L}_{2}\right)$ precipitates $\left(\gamma^{\prime}\right.$ phase) within a face-centered cubic (fcc) Ni-rich matrix ( $\gamma$ phase) [1]. A supersaturated matrix was produced by quenching a multicomponent alloy from a high temperature in a single phase field ( $\gamma$ phase) rapidly to room temperature. However, the second phase is nucleated, grow, and coarsened by diffusion of solute from the matrix towards the precipitates in an elevated temperature for sufficient atomic mobility. Nucleation is the first-order phase transition by which a metastable phase transforms into a more stable one [2]. Two important aspects for the initial phase transition in a concentrated Al-Ni system are reviewed by atom probe tomography experiments. One is the supersaturated concentration of precipitate and matrix for continuous nucleation and growth. The other is the transient volume that is not represented by the nucleated precipitates. A transient regime exists before the nucleation rate reaches its stationary value, but conventionally defines an incubation time. The metastable phase is appeared because the system initially is unstable until it reaches to critical size of precipitates. The other part of transient volume of precipitate is the interface across the interface [3].

High-purity $\mathrm{Ni}$ and $\mathrm{Al}$ were vacuum induction melted under the argon atmosphere and chill cast with a target composition of Ni-12.5 Al at. \%. Samples from the master ingot underwent an homogenization in the $\gamma$-phase field in vacuum at $1573 \mathrm{~K}$ for $20 \mathrm{~h}$ and vacancy anneal in the $\gamma$-phase field in vacuum/argon at $1223 \mathrm{~K}$ for $3 \mathrm{~h}$, followed by a direct drop-quench into water. The final stage was an aging anneal in the $\gamma+\gamma^{\prime}$ phases field at $823 \mathrm{~K}$ for times ranging from 0.08 to $4096 \mathrm{~h}$, followed by a water-quenching. This research was performed utilizing a pulsed-laser 3-D APT (a LEAP 4000X Si tomograph) and APT data were analyzed using the program IVAS 3.6.8 (Cameca, Madison, WI).

Fig.1 displays (a) the temporal evolution of the $\gamma^{\prime}$-precipitate morphology in 3-D APT reconstructions and (b) concentration profiles across the interface between $\gamma$ and $\gamma^{\prime}$ phases in the alloy aged for 0.25 and $4096 \mathrm{hrs}$. Spheroidal $\gamma^{\prime}$-precipitates are detected commencing at the earliest aging time, $0.25 \mathrm{hrs,}$ and they grow and coarsen temporally to $\mathrm{r}=14.59 \pm 1.62 \mathrm{~nm}$, where they have a cuboidal morphology, faceted on $\{100\}$ planes. And the concentration profiles from the 3-D APT results indicate that the $\gamma$ and $\gamma^{\prime}$ phases are initially supersaturated in Al from equilibrium concentration, and that the compositions of both phases evolve temporally with increasing aging time. The temporal evolution of supersaturation of compositions are Gibbs-Thomson (capillary) effect for nano-sized precipitate. The other aspect of concentration profile is the local depletion of $\mathrm{Al}$ concentration at the vicinity of interface which is controlled by the solute diffusion in in $\gamma$ matrix. Fig. 2 displays the frequency distribution of the $\mathrm{Al}$ and $\mathrm{Ni}$ concentrations with a block size of 100 atoms. The frequency distribution is created by dividing the atoms in the volume of analysis into small blocks containing a fixed number of atoms. In a small sized precipitates, the second phase is not fully separated to separated peaks. By deconvolution a distributions into three peaks, the measured $\mathrm{Ni}$ and $\mathrm{Al}$ concentration's frequncy distribution divided into matrix, precipitates and transient volume between two $\gamma+\gamma^{\prime}$ phases [4]. 


\section{References:}

[1] R.C. Reed. The superalloys: Fundamentals and applications, (Cambridge University Press, Cambridge, UK).

[2] R. Wagner, R. Kampmann, P.W. Voorhees, in "Homogeneous second-phase precipitation", ed. G. Kostorz, (Wiley-VCH; New York) p. 309.

[3] E.Y. Plotnikov et al, Scripta Mater. 70 (2014), p.51

[4] This research was supported by the National Science Foundation (NSF), Division of Materials Research (DMR-1207539). The atom-probe tomography measurements were performed at the Northwestern University Center for Atom-Probe Tomography (NUCAPT). The LEAP 4000X-Si tomograph was purchased and upgraded with funding from NSF-MRI (DMR-0420532) and ONRDURIP (N00014-0400798, N00014- 0610539, N00014-0910781).
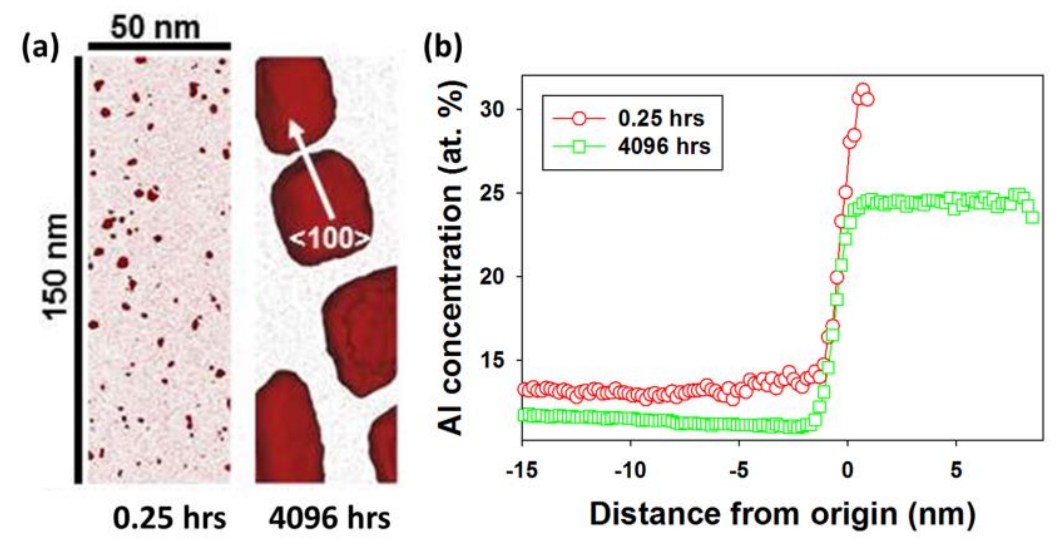

Figure 1. Temporal evolution of the $\gamma^{\prime}$-precipitate morphology in 3-D APT reconstructions and (b) concentration profiles across the interface between $\gamma$ and $\gamma^{\prime}$ phases in the alloy aged for 0.25 and 4096 hrs.

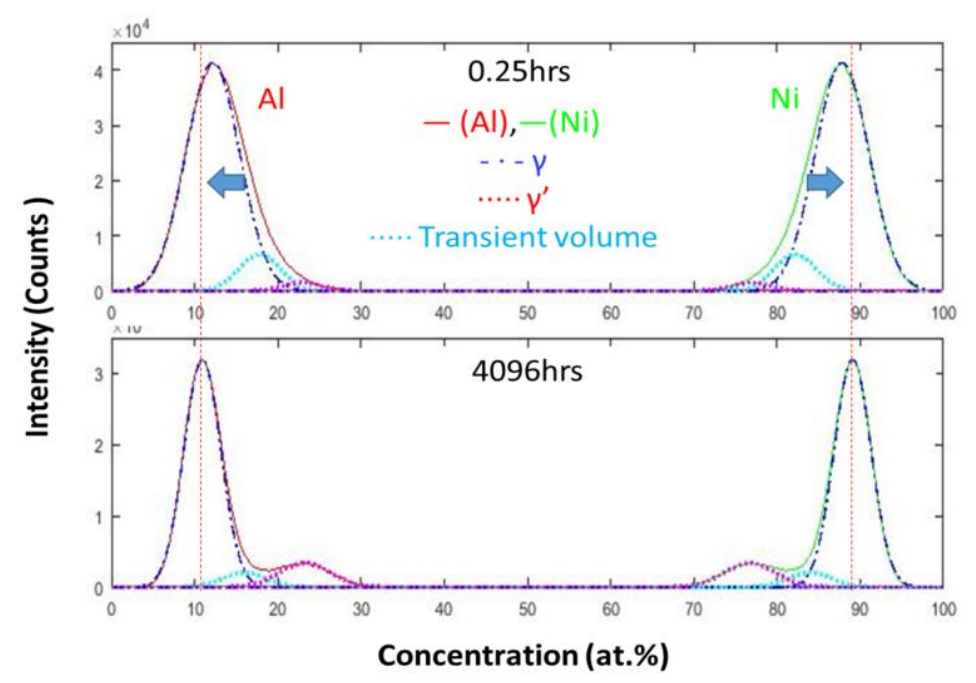

Figure 2. Frequency distribution of the $\mathrm{Al}$ and $\mathrm{Ni}$ concentrations with a block size of 100 atoms. The measured $\mathrm{Ni}$ and Al concentration's frequency distribution divided into matrix, precipitates and transient volume between two $\gamma+\gamma^{\prime}$ phases by deconvolution a detected peak into three peaks. 